\title{
The facilitation of memory performance
}

\author{
MARK PALMISANO and DOUGLAS HERRMANN \\ The U.S. Bureau of Labor Statistics, Washington, D.C.
}

\begin{abstract}
A less recognized approach to memory investigation attempts to facilitate the performance of memory tasks by producing transient improvements in an individual's capability to acquire, retain, and remember information or events. However, the lack of attention to memory facilitation should not imply that it is an unimportant topic. Memory-facilitation research is theoretically important because it elucidates not only the mechanisms and memory processes underlying facilitation but also the boundary conditions for permanent improvement in memory processing. This article explores the theoretical bases of the facilitation of memory performance.
\end{abstract}

For a variety of reasons, the study of memory has not focused on the facilitation of memory performance-that is, the transient improvement in an individual's capacity to acquire, retain, and remember events. However, the lack of attention to memory facilitation should not suggest that it is unimportant. In fact, a primary goal in many applied situations is memory facilitation. This goal is especially important when someone needs to get another person to learn something but does not need the learner to acquire and retain the facilitating process. Administrators, instructors, supervisors, and teachers frequently have such facilitation goals.

Facilitation also is important when someone needs to get another person to remember something that he or she presumably already knows. For example, government agencies gather information about the economy by asking people to recall such facts as how many hours they worked in a particular time period, how long they have been out of work, or how many and what kinds of purchases they made in a designated time period (Palmisano, 1989).

It is sometimes difficult to determine when memory performance represents facilitation, improvement, or both of these processes. Although memory improvement involves a relatively permanent alteration in memory processes, facilitation involves only a transient alteration in these processes (Ericsson, 1985). Although facilitation and improvement may be distinguished from one another in the abstract, many situations involve them both. For example, suppose someone is told to rehearse a phone number only once in a certain way to facilitate learning it. By using the recommended facilitation strategy, the person may learn the number faster than he or she normally would. However, later, when required to learn another

We gratefully acknowledge the assistance of $M$. Catharine Puskar for her comments on and help in preparing this article. Requests for reprints should be sent to Mark Palmisano, Senior Research Psychologist, U.S. Bureau of Labor Statistics, Office of Research and Evaluation, Room 2126, General Accounting Office, 441 G St. N.W., Washington, DC 20212. number, the person may revert to his or her normal learning processes.

Alternatively, the person may make use of the earlier facilitation instruction, thus demonstrating an improved memory skill. Consequently, it must be acknowledged that sometimes memory improvement may result from an attempt to facilitate memory performance. However, the research literature on this topic indicates that such results are highly unlikely. For example, even intensive memoryimprovement courses (in some cases lasting weeks) do not result in the students' permanent use of the mental manipulations that were taught to them (Herrmann \& Searleman, 1990, in press).

This article briefly reviews memory-facilitation findings reported in the research literature and then discusses the theoretical perspective on memory suggested by the findings. Generally, the literature indicates that there are essentially two types of facilitation: (1) the optimization of memory processing (presenting instructions, providing appropriate prior performance, and making use of external cues) and (2) the optimization of nonmemory factors that influence memory processing (such as physical and emotional states).

\section{The Optimization of Memory Processing}

The performance of memory tasks may be facilitated by the use of instructions, prior processing, and external cues that stimulate the transient activation of internal processes that enhance an individual's memory performance. It will be seen that research has often sought to facilitate, rather than improve, memory performance through the use of these techniques.

Instructions. Instructions may facilitate memory performance by providing a person with information about the nature of the material to be learned or remembered or by orienting the person to the best sequence in which to learn or remember the material or parts of a task. Better memory performance may be achieved temporarily by instructing a person to mentally manipulate information or experience in a particular way. Many kinds of mental manipulations have been extensively investigated and 
reported in the literature, such as mnemonics, chunking, and strategies that elaborate or reduce information to assist learning and retrieval. Generally, mental manipulations have been devised for use in the development of permanent memory skills. However, the vast amount of memory-training literature indicates that memory training has not imparted the desired sustained improvement (Herrmann \& Searleman, 1990) but instead has had the effect of facilitating memory performance rather than improving it.

Prior processing. People may temporarily optimize memory performance by processing certain information before performing the memory task of interest. The warmup is an example of such a prior processing technique. Warming up apparently has a facilitating effect on learning because exposure to certain materials temporarily activates task-specific information stored in memory. Another example is a procedure that provides a person with a mental set that gives direction and purpose to the kind of learning and remembering required by a particular memory task (Shuell, 1983).

Since it apparently enables access to memory information that otherwise would have remained inaccessible, prior processing also has a facilitating effect on retrieval. In addition, two or more presentations increase retention and promote overlearning (Farr, 1986). Also, it has long been generally accepted that distributed study improves acquisition and retention. Thus, it may be seen that prior processing may be provided by warming up, repetition, and an appropriate amount of distributed rehearsal.

Although memory training may get people to increase their use of these procedures, warming up, directions to rehearse, or distribution of study trials, when used by teachers or instructors, are intended simply to facilitate memory. It is, however, doubtful that engaging in these activities disposes people to permanently warm up more, rehearse more, or distribute their study trials differently than they did before participating in the situation in which the facilitating procedure was used.

External cues. Better memory performance may be achieved temporarily by instructing a person to make use of external cues. Virtually every normal adult assists memory with nontechnical memory aids, such as making notes or placing objects in conspicuous places as reminders (Intons-Peterson \& Newsome, in press). An important reason why external aids facilitate memory is that the physical presence of an object usually stimulates memory more effectively than imagining or thinking about the object. Because of the superior capability of physically present objects to stimulate memory, external aids have been developed to serve diverse memory functions (Herrmann \& Searleman, 1990), for example, devices to find lost objects (such as one's car keys at home or a car in a parking lot) or to remind you to do things (such as to keep an appointment or to water plants). Also, information delivery systems (such as computer-based instruction or training) that present information to be learned at optimal exposure durations, at optimal interstimulus intervals, and in perceptually salient displays, facilitate the performance of memory tasks in the classroom (Stolurow, 1969). The benefit of using such a system is that it allows the information presented to the student to be structured in a particular fashion. External aids and information delivery systems clearly facilitate memory without permanently improving memory skills.

\section{Optimization of Nonmemory Factors}

There are at least three ways that nonmemory factors may affect memory performance. One way is by influencing arousal, which, in turn, affects a person's memory performance. Another way is by influencing a person's disposition to selectively attend to a memory task. Yet another way is by influencing neurochemical processes that directly affect acquisition, retention, and/or retrieval.

Physical state. A less-than-optimal physical state may impair memory (Herrmann \& Searleman, 1990). This impairment apparently occurs because a diminished physical state reduces a person's arousal level and capacity to selectively pay attention. For example, insufficient sleep, poor nutrition, or poor physical fitness may impair memory performance. Although a good night's sleep, a nutritious meal, and moderate exercise may facilitate memory performance on a particular occasion, such facilitation will end as soon as sleeping, eating, or exercising become inadequate.

Emotional state. Memory performance in everyday life may be impaired to varying degrees by stress (Reason, 1988). The removal of stressors or the reduction of their influence on the individual (e.g., either through stress reduction or other techniques such as meditation, yoga, or exercise) can be expected to facilitate the performance of memory tasks. Similarly, retrieval may be impaired if a person's mood during retrieval is different from his or her mood during encoding. Also, people are not likely to attempt a memory task, or perform it well, if they believe that they typically fail at this task. Hence, memory performance also may be improved by changing a person's attitudes about a task.

Environmental conditions. Decrements in human motor and cognitive performance have been associated with environmental extremes and are well documented. Extremely cold and wet environments have been shown to produce physiological stress and fatigue that lead to decrements in both short-term and long-term memory, as well as in response accuracy. Similarly, extremely hot temperatures also impair memory (Wyon, Anderson, \& Lundqvist, 1979). Clearly, memory performance will be facilitated by moving people away from extreme environmental conditions. Obviously, a change in climate does not induce permanent improvement in memory skills.

Neurochemical agents. Numerous neurochemical agents impair memory: alcohol, nicotine, caffeine, marijuana, and medications such as tranquilizers and antidepressants (reviewed in Herrmann \& Searleman, 1990). 
A cessation in the use of such agents does not result in permanent memory improvement but does facilitate memory performance.

Social environment. A person's inclination to register and remember information depends on how the information meets his or her social goals (Wyer \& Srull, 1989). Thus, memory performance may be facilitated by fostering a student's awareness of an instructor's expertise and by increasing the rapport between them. Also, information will be learned and remembered better if it is consistent with the student's beliefs and cultural values.

\section{Discussion}

This article has reviewed a wide range of findings that demonstrate that performance can be temporarily enhanced by optimizing memory processes and nonmemory factors that affect memory processes. The findings reviewed here are consistent with the conclusion that facilitation procedures typically produce only transient changes in memory processing.

Facilitation findings appear to be best explained by the hypothesis that memory performance is optimized both by variables identified with the memory system (e.g., rehearsal and presentation rate) and also by variables identified with other processing modes of the psychological system (e.g., physiological, perceptual, motoric, emotional, motivational, and social). Such a multimodal model has recently been proposed to account for the effectiveness of many new procedures for improving memory ability (Herrmann \& Searleman, 1990, in press). The multimodal model can be extended to memory facilitation, first, by assuming that subjects may put their mental manipulations under the control of someone else (such as a teacher, instructor, or a friend). Second, the model can be extended to facilitation by having the person who attempts to achieve facilitation (e.g., the teacher, instructor, or a friend) affect the learner/rememberer's level of arousal by manipulating other psychological processing modes (e.g., physical state, emotional state, mood, attitudes, and social interaction) in ways that produce better memory processing. However, any model of memory facilitation must be different theoretically from models of memory improvement. We propose that memory improvement is based on the acquisition of both the habitual use of facilitative behaviors and an appropriate belief system (Pressley, Borkowski, \& Schneider, 1987) and that facilitation is not. Facilitation procedures do not endeavor to produce the habitual memory processing and appropriate belief system necessary to memory improvement. Thus, by defining the conditions under which memory processing is enhanced temporarily, facilitation research helps to identify the boundary conditions for permanent improvement in memory processing.

\section{REFERENCES}

Ericsson, K. A. (1985). Memory skill. Canadian Journal of Psychology, 39, 188-231.

FARR, M. (1986). The long-term retention of knowledge and skills. (Log No. HQ 86-31217). Alexandria, VA: Institute for Defense Analysis. Herrmann, D. J., Searleman, A. (1990). A multimodal approach to memory improvement. In G. Bower (Ed.), Psychology of learning and motivation: Advances in research and theory (Vol. 24, pp. 175-205). New York: Academic Press.

herrmann, D. J., \& Searleman, A. (in press). Memory improvement and memory theory in historical perspective. In D. Herrmann, $\mathbf{H}$. Weingartner, A. Searleman, \& C. McEvoy (Eds.), Memory improvement: Implications for memory theory. New York: Springer-Verlag.

Intons-Peterson, M. J., \& Newsome, G. L., III (in press). External memory aids: Effects and effectiveness. In D. Herrmann, H. Weingartner, A. Searleman, \& C. McEvoy (Eds.), Memory improvement: Implications for memory theory. New York: Springer-Verlag.

Palmisano, M. (1989). Understanding key labor force concepts used in the current population survey (CPS). In Proceedings of the Joint Statistical Meetings (149th Annual Meeting of the American Statistical Association), Washington, DC.

Pressley, M., Borkowskı, J. G., \& Schneider, W. (1987). Good strategy users coordinate metacognition, strategy use and knowledge. In R. Vasta \& G. Whitehurst (Eds.), Annals of child development (Vol. 4, pp. 89-129). Greenwich, CT: JAI Press.

Reason, J. T. (1988). Human error. Cambridge: Cambridge University Press.

Shuell, T. J. (1983). The effect of instructions to organize for good and poor learners. Intelligence, 7, 271-286.

STOLUROw, L. M. (1969). Instructional strategies in computer assisted instruction. (Rep. No. T44709, p. 28). Alexandria, VA: Defense Technical Information Center.

WYER, R., JR., \& SRULL, T. (1989). Memory and cognition in its social context. Hillsdale, $\mathrm{NJ}$ : Erlbaum.

Wyon, D. P., Anderson, B., Lundevist, G. R. (1979). The effects of moderate heat stress on mental performance. Scandinavian Journal of Work Environment \& Health, 5, 352-361.

(Manuscript received May 16, 1991.) 